\title{
Absolute Stabilität: Das Prinzip der Kompressionsplatte
}

\author{
- Susanne Bäuerle, Sandra Hillebrecht
}

Basiswissen

Lernziel:

1. Wie wird interfragmentäre Kompression mittels einer Platte erreicht?

2. Nenne Indikationen für eine Kompressionsplattenosteosynthese!

3. Beschreibe OP-Verlauf mit einer Platte für eine distale Humerusschaftfraktur!

4. Welche anatomische Struktur braucht besondere Vorsicht beim Zugang einer Humerusfraktur?

Ziel jeder operativen Frakturbehandlung ist die Wiederherstellung der früheren, vollen Funktion der betroffenen Extremität. Wird eine Fraktur so stabilisiert, dass im Frakturbereich unter dynamischer und funktioneller Beanspruchung kaum noch Bewegung stattfinden kann, spricht man von absoluter Stabilität.

Eine Möglichkeit, um absolute Stabilität zu erreichen, ist die Osteosynthese mit einer Platte, die als sog. „Kompressionsplatte“ verwendet wird. Interfragmentäre Kompression wird durch exzentrische Schraubenplatzierung erzeugt. Die Kompression im Frakturspalt kann zusätzlich durch eine Zugschraube durch die Platte, Plattenüberbiegung und/oder Einsatz des Plattenspanners verstärkt werden.

Bei der Kompressionsplattenosteosynthese wird die anatomische Reposition der Fragmente angestrebt, um eine Fehlstellung auszuschließen.

\section{Implantate (Abb.1)}

Platten, wie die DCP, LC-DCP und LCP können als Kompressionsplatten eingesetzt werden, da sie ein spezielles Lochdesign haben, das neutrale und exzentrische Schraubenplatzierung ermöglicht. Es müssen immer die korrespondierenden Bohrbüchsen verwendet werden.

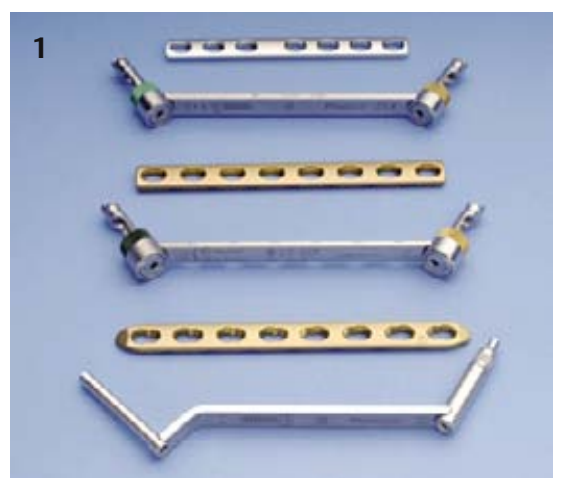

Anwendungstechnik

- Neutralbohrung und Schraubeneinbringung (Abb.2).

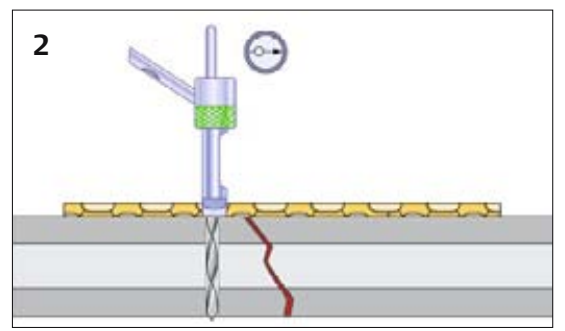

Exzentrische Bohrung (Abb.3).

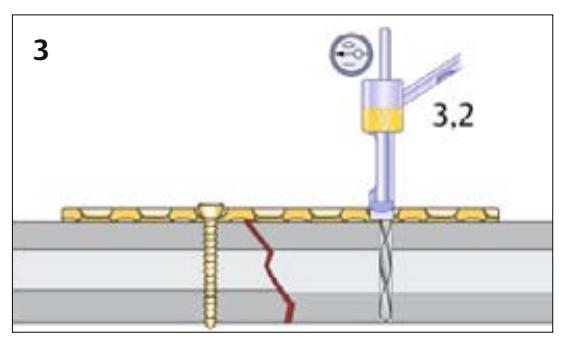

Das Festziehen der Schraube führt zur Verschiebung des Knochens und zur Kompression im Frakturspalt. (Abb.4).

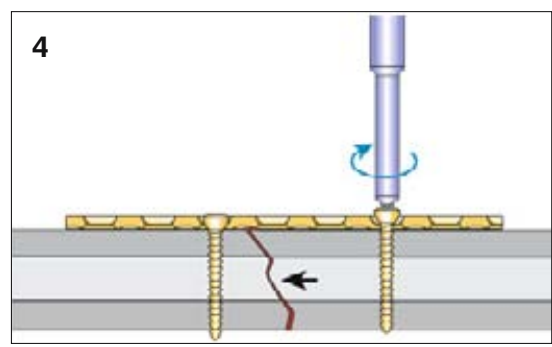

Weitere Optionen, um die interfragmentäre Kompression zu erhöhen, sind 1. Kompression durch Zugschraube

- Es besteht zusätzlich die Möglichkeit über ein Plattenloch, eine Zugschraube einzubringen (Abb.5).

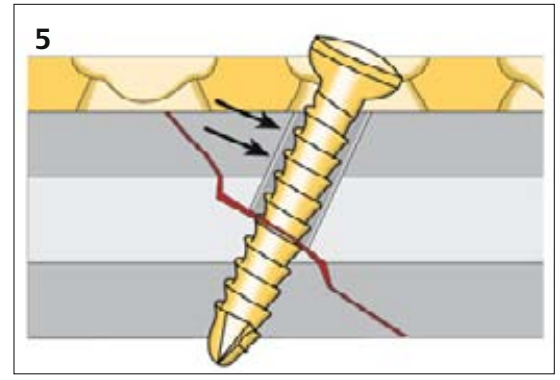

2. Kompression durch Überbiegen der Platte

- Eine Platte kann überbogen werden um mehr Stabilität in der Gegenkortikalis zu erzielen (Abb.6).

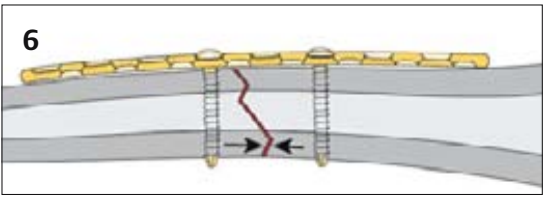

3. Kompression durch Plattenspanner - Mit Hilfe des Plattenspanners kann eine Verschiebung des Knochens im Frakturspalt um 1-2 mm erreicht werden (Abb. 7).

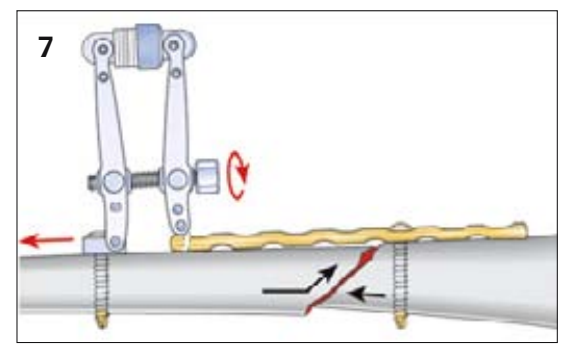


Indikationen für Kompressionsplattenosteosynthese

- Humerusschaftfraktur

- Unterarmschaftfraktur (Ulna, Radius)

- Korrekturosteotomien

Technik der Kompressionsplattenosteosynthese bei distaler Humerusschaftfraktur (Abb.8)

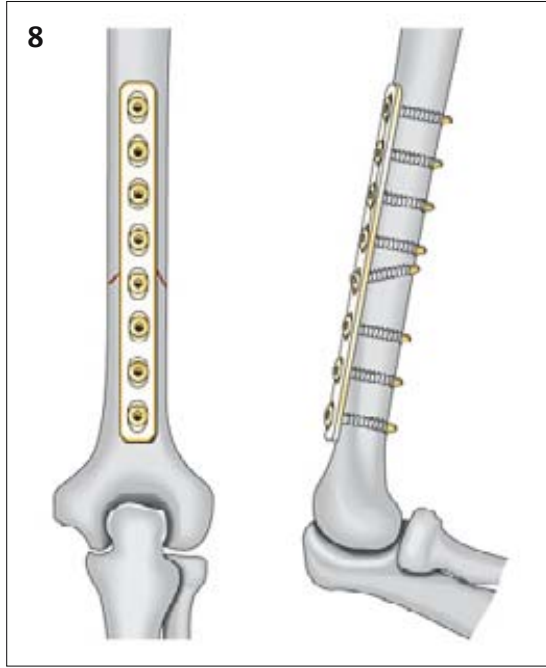

1. Bauchlage, Arm auf Armbänkchen, $90^{\circ}$ Stellung hängend und freibeweglich lagern (Abb.9).

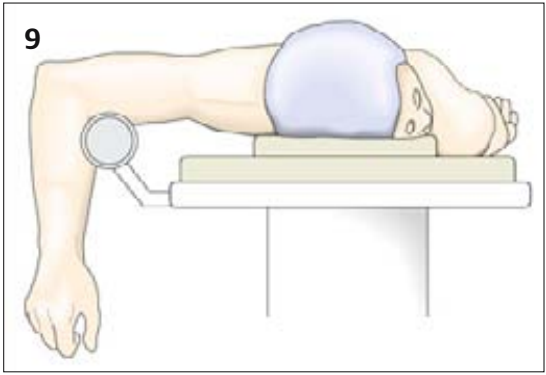

2. Dorsaler Zugang (Abb. 10).

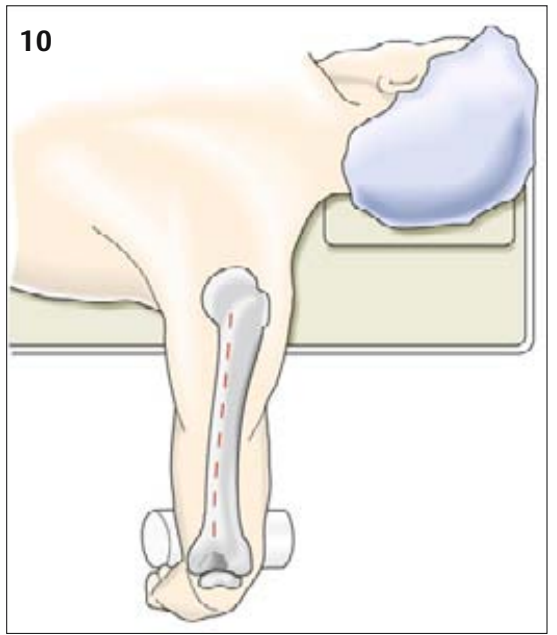

3. Aufsuchen und Anschlingen des Nervus radialis (Abb.11).

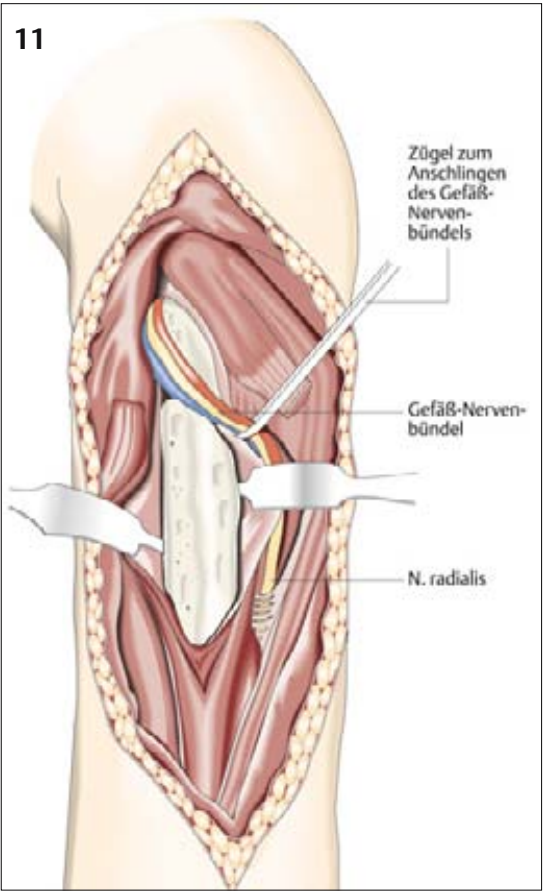

4. Fraktur reponieren

5. Anpassen (Biegeschablone) einer schmalen oder breiten Großfragment-LC DCP (alternativ DCP, LCP) und Zurechtbiegen mit der großen Biegepresse

6. Platte vorsichtig unter den Nervus. radialis schieben und an den Knochen anlegen

7. Setzen des ersten Bohrloches nahe der Fraktur mit Spiralbohrer 3,2 mm und der entsprechenden Bohrbüchse in Neutralstellung (grün, Pfeil frakturweisend)

8. Bestimmen der Schraubenlänge mit dem Tiefenmessgerät

9. Gewindeschneiden $4,5 \mathrm{~mm}$ mit entsprechender Gewebeschutzhülse. Alternative: selbstschneidende Schrauben verwenden.

10. Einbringen der Kortikalisschraube 4,5, wahlweise mit Antriebsmaschine oder manuell

11. Setzen des zweiten Bohrloches im gegenüberliegenden Fragment mit dem Spiralbohrer 3,2 $\mathrm{mm}$ und der entsprechenden Bohrbüchse 3,2 mm in Kompressionsstellung (gelb, Pfeil frakturweisend)

12. Längenmessung

13. Gewindeschneiden

14. Einbringen der Schraube

15. Besetzen der restlichen Schraubenlöcher in Neutralstellung.

16. Röntgenabschlusskontrolle in 2 Ebenen

\section{Instrumentarium}

Anordnung der Instrumente für Kompressionsplattenosteosynthese mit einer LC DCP 4,5 mm (Abb. 12).

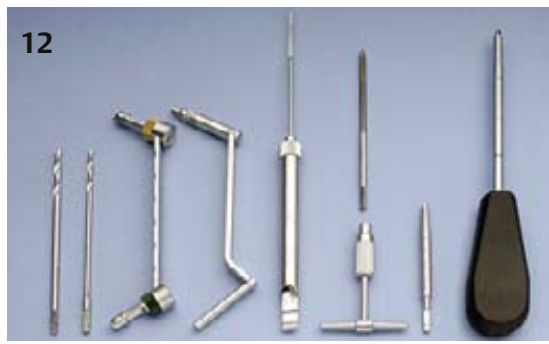

Anordnung der Instrumente zum Biegen der Platte (Abb.13).

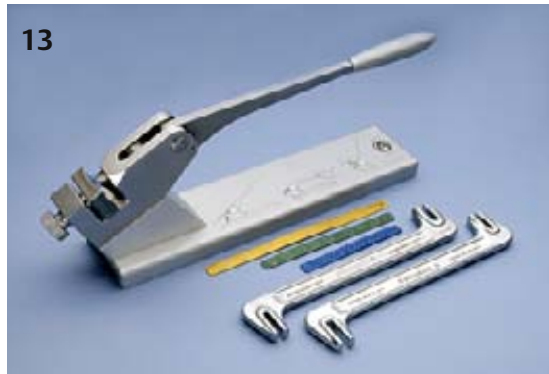

Anordnung der Instrumente zum Plattenspannen

- Plattenspanner (Abb. 14).

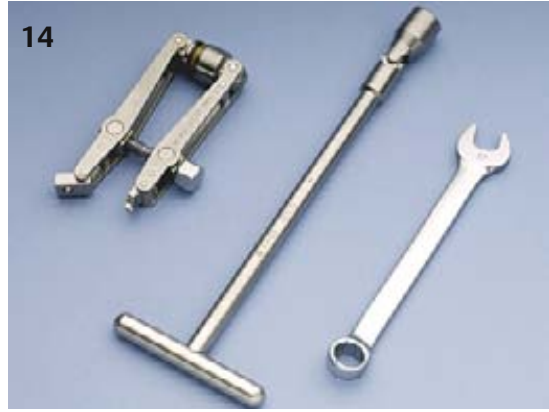

Nicht vergessen

Zügel zum Anschlingen des Nervus radialis

\section{Susanne Bäuerle}

Direktorin ORP/Nurse Education

AO International

Clavadelerstrasse 8

7270 Davos

\section{Sandra Hillebrecht}

Operations Technische Assistentin

OSK St. Elisabeth

OP-Abteilung Unfallchirurgie mit Sektion Orthopädie, Elisabethenstraße 15 88212 Ravensburg 